\title{
Direct-acting antiviral treatments display excellent outcomes even in older HCV-infected patients at increased risk of fibrosis
}

\author{
Huan Xia ${ }^{1,2 \#}$, Yaping Zhang ${ }^{3 \#}$, Silvere D. Zaongo ${ }^{1}$, Jing Liang ${ }^{4}$, Xiaowen Gong ${ }^{5}$, Yue $\mathrm{Hu}^{1}$, Ping Ma ${ }^{1,2}$, \\ Fengmei Wang ${ }^{4}$
}

${ }^{1}$ Department of Infectious Diseases, Tianjin Second People's Hospital, Tianjin, China; ${ }^{2}$ Tianjin Association of STD/AIDS Prevention and Control, Tianjin, China; ${ }^{3}$ The Third Central Clinical College of Tianjin Medical University, Tianjin, China; ${ }^{4}$ Department of Gastroenterology and Hepatology, Tianjin Third Central Hospital, Tianjin, China; ${ }^{5}$ State Key Laboratory of Experimental Hematology, National Clinical Research Centre for Blood Diseases, Institute of Hematology and Blood Diseases Hospital, Chinese Academy of Medical Sciences and Peking Union Medical College, Tianjin, China

Contributions: (I) Conception and design: F Wang; (II) Administrative support: F Wang, P Ma; (III) Provision of study materials or patients: F Wang, J Liang, P Ma; (IV) Collection and assembly of data: H Xia, Y Zhang, J Liang, Y Hu, P Ma; (V) Data analysis and interpretation: H Xia, SD Zaongo, X Gong; (VI) Manuscript writing: All authors; (VII) Final approval of manuscript: All authors.

"These authors contributed equally to this work.

Correspondence to: Fengmei Wang. Department of Gastroenterology and Hepatology, Tianjin Third Central Hospital, Tianjin 300170 , China. Email: wangfengmei@tmu.edu.cn; Ping Ma. Department of Infectious Diseases, Tianjin Second People's Hospital, Tianjin 300192, China. Email: mapingtianjin@163.com.

Background: This study compared the efficacy and tolerability of available direct-acting antiviral (DAA) regimens between individuals aged 60 years and older and younger patients in a real-life setting. Specifically, we aimed to provide evidence of the efficacy and safety of DAAs in the treatment of older adults in Tianjin, China.

Methods: In this retrospective observational cohort study, patients with chronic hepatitis C virus (HCV) were enrolled between April 2018 and December 2019 at 2 tertiary hospitals in Tianjin, China. We assessed the sustained virologic response (SVR) 12 weeks (SVR12) after DAA treatment, and adverse events in two groups using age stratification by comparing older adults ( $\geq 60$ years) and younger adults ( $<60$ years). Logistic regression analyses were performed to explore the risk factors associated with the SVR12.

Results: Of 1,106 patients, 440 (39.8\%) were $\geq 60$ years of age. The overall SVR12 rate was $97.8 \%$ in the entire cohort. In the older adult group, the SVR12 rate was 98.0\% (431/440) compared to 97.7\% (651/666) in the younger adult group. A multivariate analysis showed that (I) age was not predictive of SVR; and (II) the variables of treatment-experience [adjusted odds ratio $(\mathrm{aOR})=27.53 ; 95 \%$ confidence interval (CI) $=3.35-226.08 ; \mathrm{P}=0.002]$ and aspartate aminotransferase (AST) $(\mathrm{aOR}=1.02 ; 95 \% \mathrm{CI}=1.01-1.04 ; \mathrm{P}=0.027)$ were independently associated with the SVR12 in the older adult group. All of the available DAA regimens were well-tolerated in older adult group.

Conclusions: Chinese older adults with chronic HCV infection showed a significantly higher percentage of fibrosis; however, the available different DAA regimens were safe, well-tolerated, and achieved high rates of SVR in all age subgroups. Our observations suggest that DAA treatment should not be withheld even from older patients suffering from chronic HCV.

Keywords: Direct-acting antivirals; older adults; hepatitis C virus (HCV); efficacy; sustained viral response

Submitted Mar 04, 2021. Accepted for publication Apr 13, 2021.

doi: 10.21037/atm-21-1297

View this article at: http://dx.doi.org/10.21037/atm-21-1297 


\section{Introduction}

Affecting an estimated 71 million people worldwide and 9.8 million in China, hepatitis $\mathrm{C}$ virus (HCV) infection is a major threat to public health (1). A recent systematic review and meta-analysis showed that the overall prevalence rate of anti-HCV seropositivity among the general population in mainland China was $0.91 \%$, and varied across different regions (2). The prevalence of anti-HCV increased continuously with age, analyses stratified by age indicated that individuals $\geq 65$ years had the highest prevalence of anti-HCV (3.95\%) (2). Similarly, the reported prevalence of anti-HCV tends to be higher in the older population compared to younger persons in many other countries such as Japan, Italy, and Egypt (3). Viremic HCV tends to be more prevalent among older patients than younger patients in many countries (3), including mainland China (4). Further, older HCV-infected patients are more likely to experience chronic and advanced liver diseases, such as fibrotic progression, end-stage liver disease, and hepatocellular carcinoma (HCC) development because of the increased duration of infection $(3,5,6)$. Due to the increasing rates of side effects and the higher prevalence of concurrent comorbidities (3), interferon-based therapy in older patients frequently leads to treatment intolerance and ineligibility, which challenges the clinical applications of these therapeutic options. The advent of direct-acting antivirals (DAAs) has revolutionized the treatment landscape of $\mathrm{HCV}$ infection. As reported in several clinical trials and real-world settings (7), all-oral DAA regimens are highly effective, safe and have an excellent sustained virologic response (SVR). However, certain conditions $(8,9)$ [fibrosis status, comorbidities, treatment-naive $v s$. experienced, coinfection with human immunodeficiency virus (HIV) or hepatitis B virus (HBV), solid organ transplant recipients] and viral characteristics $(8,10)$ (genotype, resistanceassociated substitutions) may lower the SVR results.

In the current HCV guidelines (11-13), focused attention has been chosen for the panel on HCV management among special/unique populations $(11,12)$ such as adolescents and children, pregnant women, prisoners, people who inject drugs. But there are no recommendations regarding the care of the elderly population with an elevated risk of fibrosis and more comorbidities. Given the increasing age and number of Chinese older patients with $\mathrm{HCV}$ in recent years, it is critical to understand whether the application of DAA therapy could result in satisfactory virologic outcomes and/or well-tolerated treatments. To date, these questions remain unanswered, as the data are limited, which is mainly due to the fact that older patients are usually excluded or underrepresented in clinical trials (3). Further, the majority of real-world studies that have examined the treatment outcomes and safety of DAA therapy among older HCV patients have been conducted in Western countries (14-18); thus, the data for Asian countries are very limited $(19,20)$. Additionally, these studies (I) focused on only one DAA regimen $(14,21)$, (II) considered only one HCV genotype $(15,20,22)$, or (III) lacked power due to their limited sample sizes $(16,19)$.

In 2017, DAAs were officially introduced in China for the first time (23); however, access to brand DAAs remains limited due to their high costs. Tianjin was the first city to pilot a capitated payment for HCV treatment in China. Since April 2018, adult patients with chronic hepatitis C (CHC) have had access to Tianjin local government reimbursed DAA therapy with no fibrosis (FIB) stage restrictions $(23,24)$. DAA regimens include elbasvir/grazoprevir (EBR/GZR), ombitasvir/paritaprevir/ ritonavir/dasabuvir (OBV/PTV/r/DSV), sofosbuvir (SOF), daclatasvir (DCV), and asunaprevir (ASV). SOF/velpatasvir (SOF/VEL) was approved for the reimbursement list in January 2020. The aforementioned regimens are currently recommended by the latest Chinese guidelines (the 2019 version) for the treatment of hepatitis $\mathrm{C}$ (25).

Due to the local reimbursement policy and early access to DAAs, Tianjin's HCV treatment approach is significantly ahead of that of China. To date, the benefits of the response of Chinese older patients to DAA treatment has not been demonstrated. This study compared the effectiveness and safety of available DAA regimens in older and younger $\mathrm{HCV}$-infected patients. Most importantly, it closely analyzed DAA treatment and safety outcomes in older HCV patients.

We present the following article in accordance with the STROBE reporting checklist (available at http://dx.doi. org/10.21037/atm-21-1297).

\section{Methods}

\section{Study design and patients selection}

Between April 2018 and December 2019, a retrospective observational cohort study was conducted in Tianjin, China. The data concerned chronic HCV-infected patients from 2 tertiary, insurance-designated hospitals; that is, the Tianjin Second People's Hospital, and Tianjin Third Central Hospital. Almost all of the HCV patients in the Tianjin district who had been treated with DAAs and who 
were covered by local health insurance were included in the study.

To be eligible to participate in the study, patients had to meet the following inclusion criteria: (I) be an adult (aged $\geq 18$ years); (II) have a chronic HCV infection of any genotype (and subtype); (III) have FIB at any stage; (IV) be treatment naïve or experienced; (V) have Tianjin local health insurance; and (VI) have been treated with DAAs reimbursed by the Tianjin local government. Conversely, patients were excluded from the study if they met any one of the following exclusion criteria: (I) were younger than 18 years of age; (II) had a recent history of drug abuse; (III) were pregnant or breast-feeding women; (IV) had chronic liver disease of a non-HCV etiology (e.g., autoimmune hepatitis, alcoholic liver disease, Wilson's disease, or hemochromatosis); (V) had incomplete or missing data; (VI) had discontinued treatment; and/or (VII) lacked follow-up data for an SVR12 assessment.

The study was conducted in accordance with the Declaration of Helsinki (as revised in 2013). This study was reviewed and approved by the Tianjin Second People's Hospital Institutional Review Board (No. 2018-8), which is a leading research institute. Written and informed consent was provided by each of the recruited participants.

Older individuals were defined as those aged $\geq 60$ years based on the World Health Organization's criterion, (26) and the Elderly Rights Guarantees Law in China (27). Thus, participants were divided into groups based on whether they were "younger" (i.e., $<60$ years) or "older" (i.e., $\geq 60$ years). In relation to the older adult group, the participants were further divided into the following three subgroups based on the stratification of Forman et al. (28): (I) the 60-69 age group; (II) the 70-79 age group; and (III) the $\geq 80$ age group.

\section{Treatment regimens}

At the time of enrollment, the selection of the DAA regimen and the duration of treatment (12 or 24 weeks) were based on the HCV genotype and liver stage as recommended by the current guidance from the Asian-Pacific Association for the Study of the Liver (13). The following currently available reimbursed DAA regimens were included in this study: (I) EBR $(50 \mathrm{mg}) / \mathrm{GZR}(100 \mathrm{mg})$ daily; (II) OBV/PTV/r $(25 \mathrm{mg} / 150 \mathrm{mg} / 100 \mathrm{mg}$ once daily)/DSV (500 mg daily, twice a day) \pm ribavirin (RBV) daily $(1,000$ or $1,200 \mathrm{mg}$ daily divided into 3 doses in patients weighting $<75$ or $\geq 75 \mathrm{~kg}$, respectively); (III) SOF (400 mg once daily)
+ RBV daily; (IV) SOF (400 mg) + DCV (60 mg) \pm RBV daily; (V) DCV (60 mg) + ASV (100 mg) twice daily; and (VI) SOF/VEL (400 mg/100 mg) \pm RBV daily. RBV was used at the physician's discretion in accordance with the practice guidelines.

\section{Data collection}

Basic demographic characteristics, clinical data, and laboratory values were systematically collected by reviewing patients' medical records. The clinical data included DAA regimens, any history of prior treatment, liver imaging results, physical findings, concomitant comorbidities, and comedication. The laboratory data included the HCV genotype, HCV ribonucleic acid (RNA) (at baseline, the end of treatment, and week 12 after treatment completion), liver function tests (albumin, alanine aminotransferase, aspartate aminotransferase, bilirubin, and the international normalized ratio), kidney function, estimated glomerular filtration rate, a complete blood count, alpha-fetoprotein (AFP), anti-HIV antibody, HBV surface antigen, autoantibodies, and thyroid function. HCV RNA was measured using the COBAS ${ }^{\circledR}$ AmpliPrep/COBAS ${ }^{\circledR}$ TaqMan HCV Quantitative Test (Roche Molecular Systems, Branchburg, $\mathrm{NJ}$, USA; version 2.0) with a detection limit of $15 \mathrm{IU} / \mathrm{mL}$. $\mathrm{HCV}$ genotype was determined using a sequencing method.

Cirrhosis was determined by transient elastography (Fibroscan ${ }^{\circledR}$ results $\geq 14.6 \mathrm{kPa}$ ), or the presence of histological, endoscopic, radiologic, clinical evidence of cirrhosis, and/or portal hypertension (presence of varices, splenomegaly). Decompensated cirrhosis was defined as the history or presence of clinical ascites, variceal hemorrhage, and/or encephalopathy. HCC was screened by one or more imaging modalities, or by one imaging diagnostic tool and an AFP level $>400 \mathrm{ng} / \mathrm{mL}$.

\section{Effectiveness and safety analysis}

The primary efficacy endpoint was the percentage of patients achieving SVR12 [defined as HCV RNA < the lower limit of quantitation $(15 \mathrm{IU} / \mathrm{mL})$ at week 12 after the completion of therapy]. The adverse events (AEs) and serious adverse events (SAEs) were determined by combining symptoms reported by the participants with abnormal laboratory findings. All such events were recorded during and after treatment. AEs were classified in accordance with the Common Terminology Criteria (Version 5.0) as established by the National Cancer 


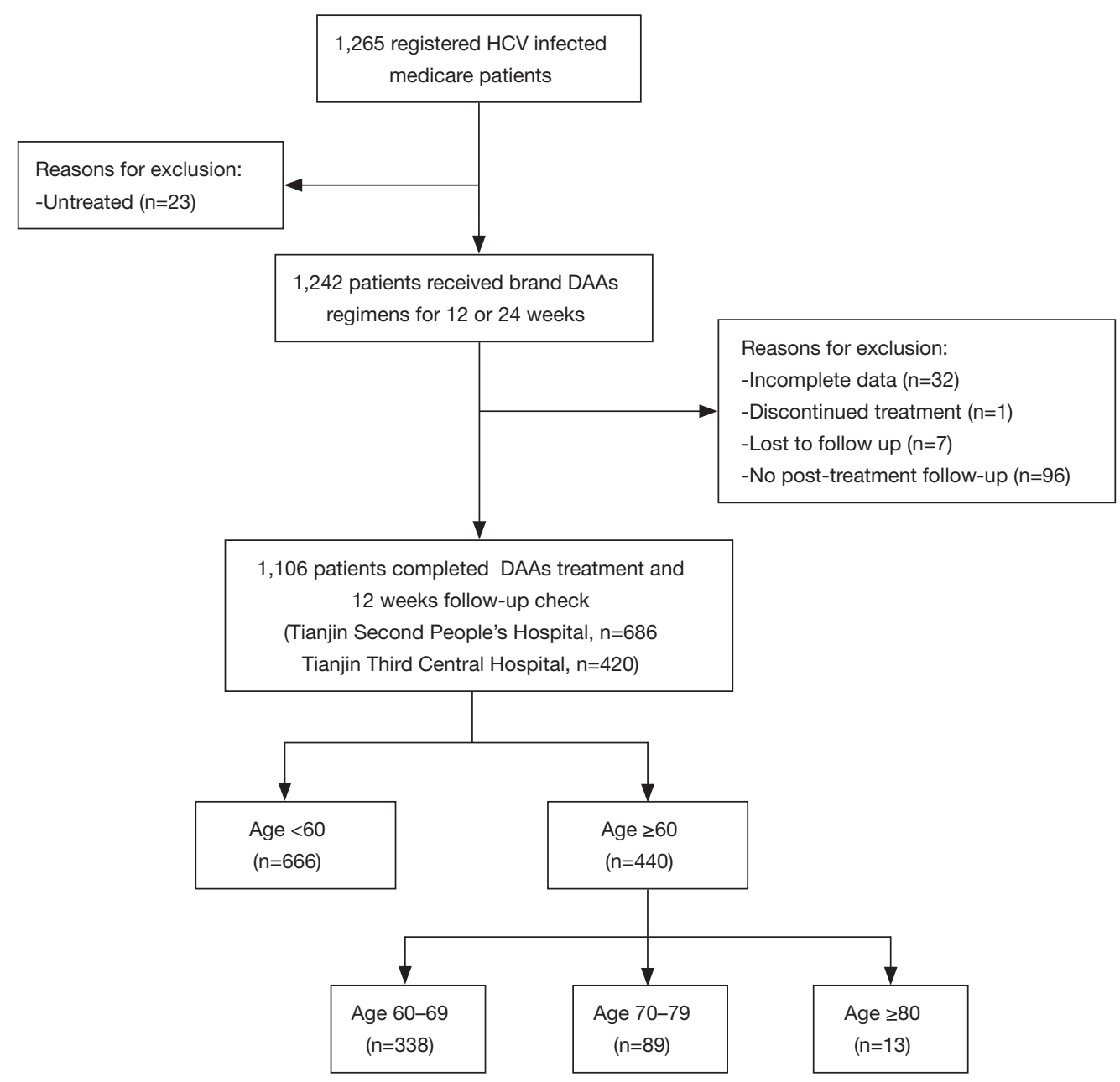

Figure 1 Flowchart of participants enrollment.

Institute (29). The severity of AEs was determined using unique clinical descriptions for each event, and graded as mild, moderate, severe, life-threatening, or death (grades $1-5$, respectively).

\section{Statistical analysis}

Categorical variables were reported as numbers and percentages; continuous variables were presented as means [standard deviations (SDs)] or medians [interquartile ranges (IQRs)] as appropriate. Differences in categorical variables were assessed using the Chi-square test or Fisher's exact test. Continuous variables were compared using $t$-tests or the Mann-Whitney $U$ test. To assess the factors associated with the SVR, univariate and multivariate stepwise regression analyses were used to calculate the odds ratios (ORs) and 95\% confidence intervals (CIs). All tests were
2 -tailed, and a $\mathrm{P}$ value $<0.05$ was considered statistically significant. Statistical analyses were carried out using SAS version 9.4 software (SAS Institute, Cary, NC, USA).

\section{Results}

Between April 2018 and December 2019, 1,265 patients were screened, of which 1,106 completed both the DAA treatment and 12-week follow-up compliance procedure. Details of the enrollment process are shown in Figure 1.

\section{Characteristics of the study population}

Overall, 1,106 patients were identified and included in the study. The demographic and clinical characteristics of the study population (as stratified by age) are illustrated in Table 1. The participants comprised 440 (39.8\%) patients in 
Table 1 Demographic and clinical features

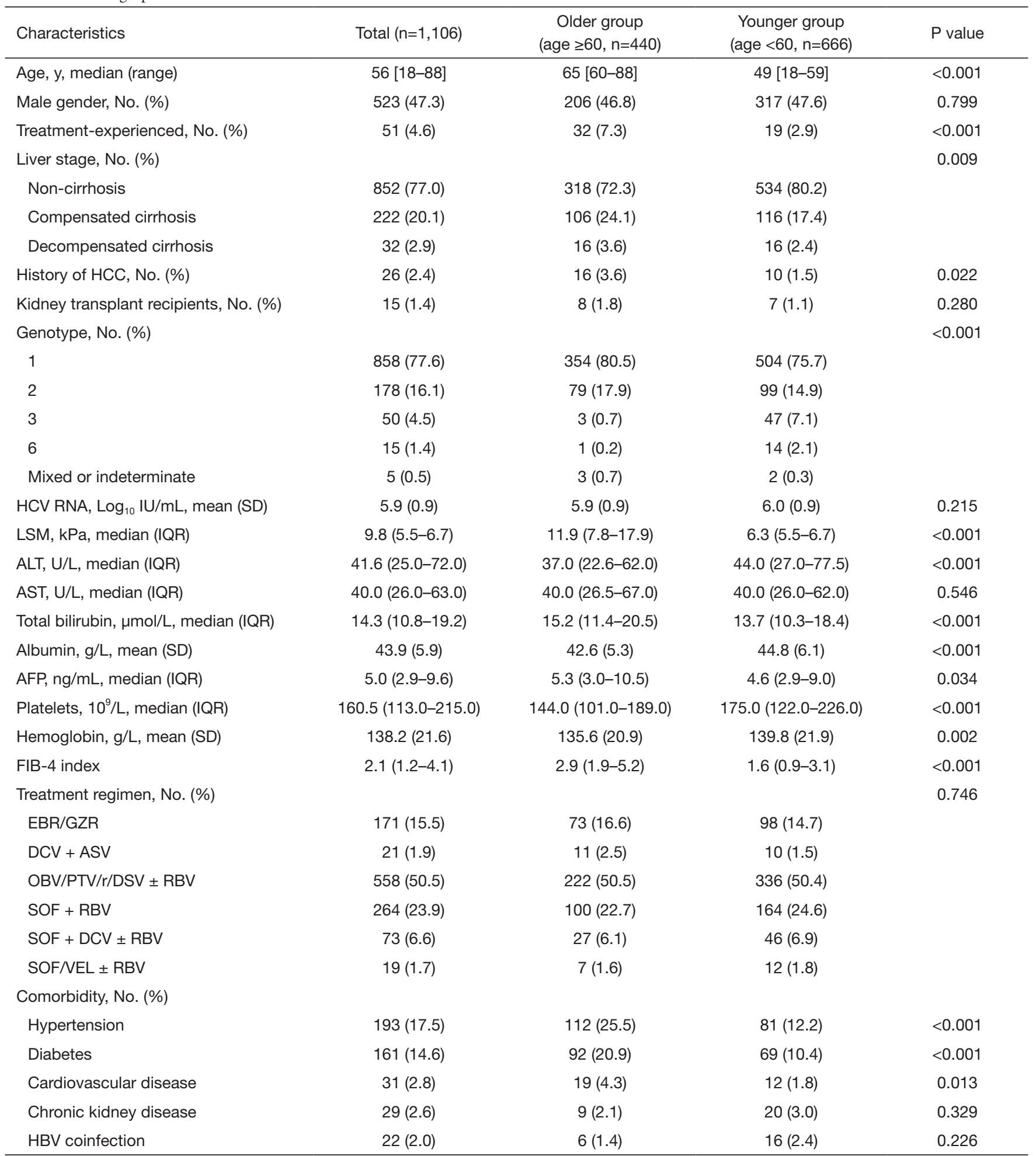

HCC, hepatocellular carcinoma; HCV, hepatitis C virus; SD, standard deviation; IQR, interquartile range; LSM, liver stiffness measurement; ALT, alanine aminotransferase; AST, aspartate aminotransferase; AFP, alpha-fetoprotein; FIB-4, Fibrosis-4; EBR, elbasvir; GZR, grazoprevir; DCV, daclatasvir; ASV, asunaprevir; OBV/PTV/r/DSV, ombitasvir/paritaprevir/ritonavir/dasabuvir; RBV, ribavirin; SOF, sofosbuvir; VEL, velpatasvir; HBV, hepatitis B virus. 
the older group ( $\geq 60$ years) and $666(60.2 \%)$ in the younger group ( $<60$ years). The median age of the study participants was 56 years (range, 18-88 years); however, patients in $<60$ years group were significantly younger than those in the older group (49 vs. 65; $\mathrm{P}<0.001$ ). Nearly half $(46.8 \%)$ of the older patients were males, and $7.3 \%$ were treatmentexperienced. The proportions of patients who were treatment-experienced or who had a history of previous HCC were significantly higher in the older group than the younger group $(\mathrm{P}<0.001$ and $\mathrm{P}=0.022$, respectively).

The proportion of patients with (I) liver cirrhosis increased with age, (II) compensated cirrhosis increased progressively from $17.4 \%$ in the younger group to $24.1 \%$ in the older group, and (III) decompensated cirrhosis was $2.4 \%$ (younger group) and 3.6\% (older group). The proportion of patients with advanced liver stages was higher in the older group than the younger one $(\mathrm{P}=0.009)$. Similarly, the liver stiffness measurement (LSM) and FIB scores (FIB-4 index) were significantly higher in the age $\geq 60$-age group (all $\mathrm{P}<0.001)$.

Most patients had genotype 1 (77.6\%). In the entire cohort, most patients $(50.5 \%)$ were treated with $\mathrm{OBV} /$ $\mathrm{PTV} / \mathrm{r} / \mathrm{DSV} \pm \mathrm{RBV}$, and the treatment regimens did not differ significantly between the two cohorts $(\mathrm{P}=0.746)$.

The 5 most frequently reported comorbidities in the entire group were hypertension (17.5\%), diabetes (14.6\%), cardiovascular disease (2.8\%), chronic kidney disease (2.6\%), and $\mathrm{HBV}$ coinfection $(2.0 \%)$. Compared to the younger group, the older group had significantly more patients with hypertension $(\mathrm{P}<0.001)$, diabetes $(\mathrm{P}<0.001)$, and cardiovascular disease $(\mathrm{P}=0.013$; see Table 1$)$.

Further, alanine aminotransferase (ALT), albumin, hemoglobin, and platelets were significantly lower for patients aged $\geq 60$ than for those aged $<60$. Conversely, total bilirubin and AFP were significantly higher for patients aged $\geq 60$ than for those aged $<60$. There was no difference between the two groups in terms of gender, HCV RNA, aspartate aminotransferase (AST), and the percentage of kidney transplant recipients.

\section{Subgroup analysis of the older patients}

As stated above, patients in older age group ( $\geq 60$ years) were further divided into 1 of the following three subgroups: (I) the 60-69 age group ( $\mathrm{n}=338)$; (II) the 70-79 age group $(\mathrm{n}=89)$; and (III) the $\geq 80$ age group ( $\mathrm{n}=13)$. The median age for patients in the older age cohort was 65 years (range w60-88), and the median ages of these patients were statistically different among the three subgroups (63, 73 , and 83 respectively, $\mathrm{P}<0.001$; see Table 2). Among the considered variables, the FIB-4 index $(\mathrm{P}=0.044)$ and hypertension $(\mathrm{P}=0.033)$ proportions increased significantly in the $60-69,70-79$, to $\geq 80$ age groups (see Table 2). Moreover, it appeared that liver stiffness was associated with older age; however, it should be noted that the subgroup comparison was not statistically significant (60-69 LSM $=11.9,70-79 \mathrm{LSM}=13.8$, and $\geq 80 \mathrm{LSM}=7.7 ; \mathrm{P}=0.082$ ). The results showed that participants in the advanced older age group displayed more severe liver FIB. The exception of those in the $\geq 80$ subgroup with LSM $=7.7$ could be explained by the very limited size of the cohort $(n=13)$. Finally, no significant difference was noted between the groups in relation to gender, previous treatment history, liver stage, history of HCC, type of genotype, baseline HCV RNA, ALT, AST, AFP, total bilirubin, albumin, platelet, hemoglobin, treatment regimen, and comorbidities (see Table 2).

\section{Virologic response}

Figure 2 shows the SVR12 rates of the entire study group as stratified by age, HCV genotype, treatment regimens, liver stage, and previous treatment history in the older group. The overall rate of SVR in the entire cohort was $97.8 \%$. In each of the age subgroups of $60-69,70-79$, and $\geq 80$, the percentage of patients achieving SVR12 was $98.2 \%, 96.6 \%$, and $100 \%$, respectively (see Figure $2 A$ ). The SVR12 response rates were relatively similar in the older and younger groups (98.0\% vs. $97.7 \%)$. In analyzing $\mathrm{HCV}$ genotypes, we noted that the SVR rates for all genotypes were superior to $97 \%$ (see Figure 2B). Further, all of the DAA regimens had an extremely high SVR rate $(\geq 96 \%$; see Figure $2 C$ for further details). Finally, in relation to liver stage, (I) a favorable treatment response was observed in patients with CHC (99.4\%), (II) suboptimal SVR12 rates were reported in patients with compensated cirrhosis (94.3\%), and (III) decompensated cirrhotic individuals displayed the lowest SVR (93.8\%). In addition, HCV treatment-experienced patients $(87.5 \%)$ had significantly lower rates of SVR than treatment-naïve patients $(98.8 \%$; see Figure 2D).

\section{Factors associated with SVR12}

A logistic regression analysis was used to identify the risk factors associated with SVR12 in older patients. The results 
Table 2 Subgroup analysis of older patients (age $\geq 60$ years)

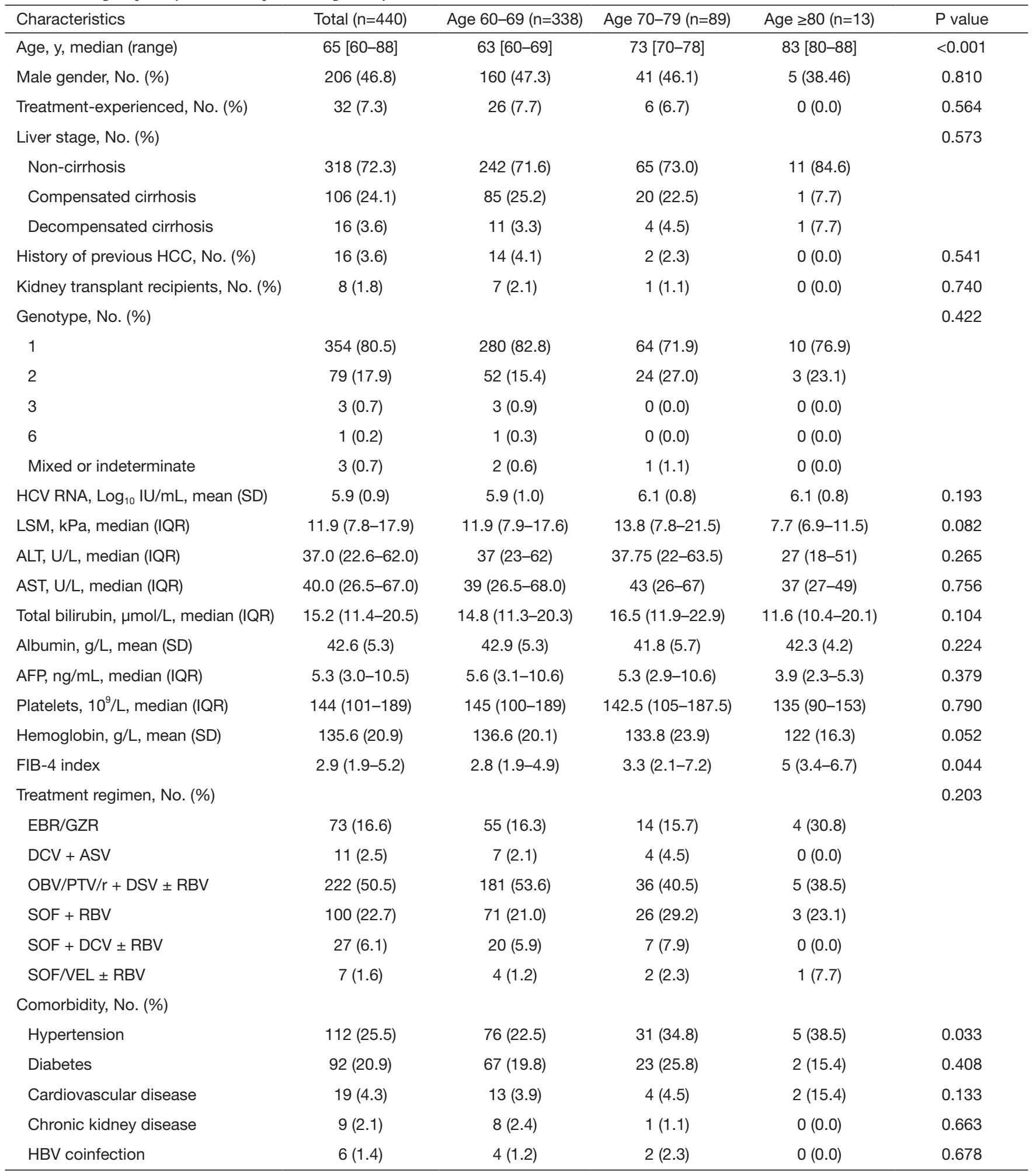

HCC, hepatocellular carcinoma; HCV, hepatitis C virus; SD, standard deviation; IQR, interquartile range; LSM, liver stiffness measurement; ALT, alanine aminotransferase; AST, aspartate aminotransferase; AFP, alpha-fetoprotein; FIB-4, Fibrosis-4; EBR, elbasvir; GZR, grazoprevir; DCV, daclatasvir; ASV, asunaprevir; OBV/PTV/r/DSV, ombitasvir/paritaprevir/ritonavir/dasabuvir; RBV, ribavirin; SOF, sofosbuvir; VEL, velpatasvir; HBV, hepatitis $B$ virus. 

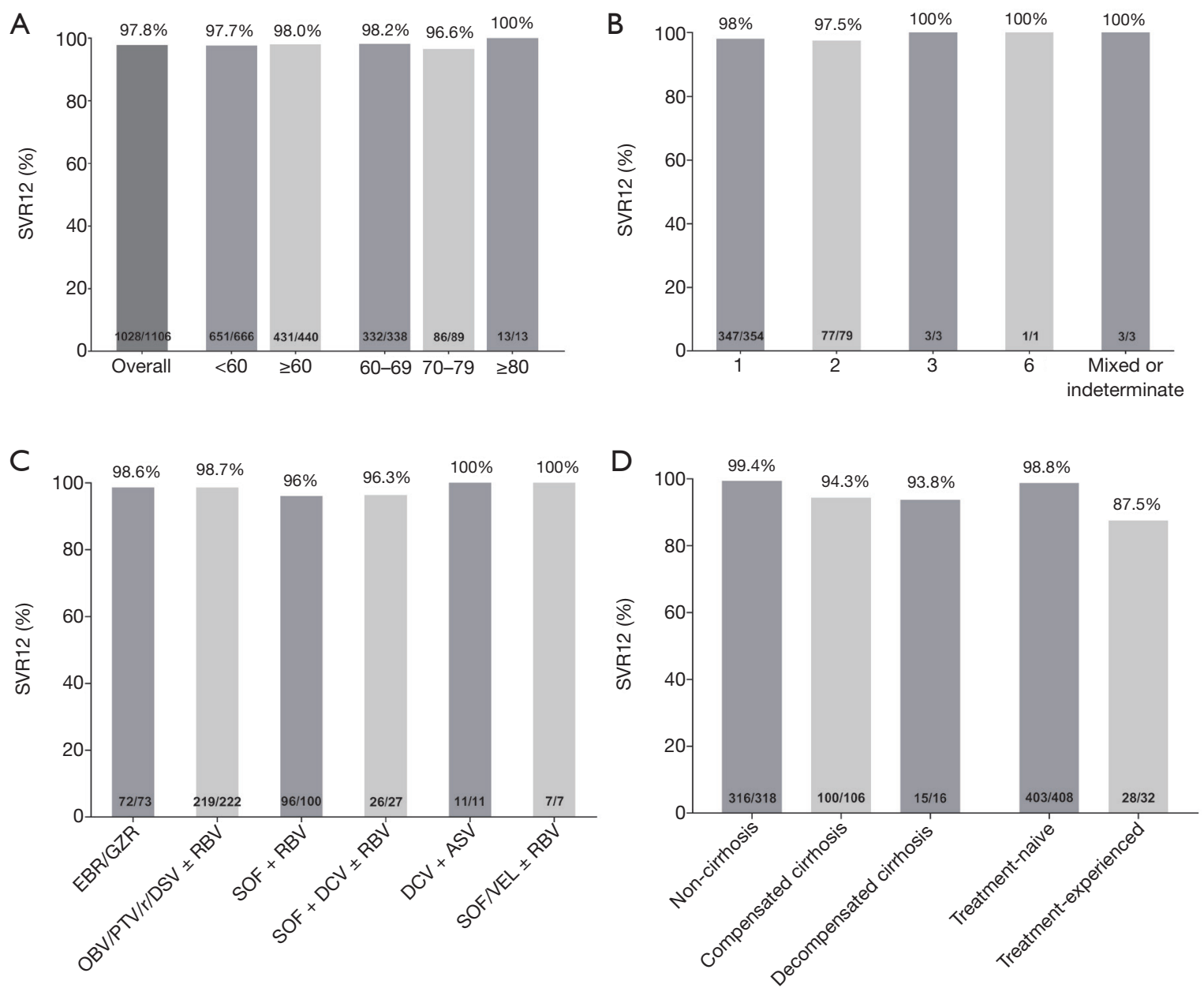

Figure 2 Sustained virologic response at week 12 post-treatment (SVR12) in the study population based on (A) age; (B) genotypes; (C) treatment regimen; and (D) liver stage and treatment history. EBR, elbasvir; GZR, grazoprevir; OBV/PTV/r/DSV, ombitasvir/paritaprevir/ ritonavir/dasabuvir; RBV, ribavirin; SOF, sofosbuvir; DCV, daclatasvir; ASV, asunaprevir; VEL, velpatasvir.

of this analysis are presented in Table 3. In the univariate analysis, treatment-experienced patients $(\mathrm{OR}=11.51 ; 95 \%$ $\mathrm{CI}=2.93-45.29 ; \mathrm{P}<0.001)$, compensated cirrhosis $(\mathrm{OR}$ $=9.48 ; 95 \% \mathrm{CI}=1.88-47.72 ; \mathrm{P}=0.006)$, and a history of $\operatorname{HCC}(\mathrm{OR}=8.51 ; 95 \% \mathrm{CI}=1.62-44.72 ; \mathrm{P}=0.011)$ were associated with the SVR12. In the multivariable stepwise regression model, treatment-experience (adjusted odds ratio $(\mathrm{aOR}=27.53 ; 95 \% \mathrm{CI}=3.35-226.08 ; \mathrm{P}=0.002)$ and AST $(\mathrm{aOR}=1.02 ; 95 \% \mathrm{CI}=1.01-1.04 ; \mathrm{P}=0.027)$ were associated with SVR12 while controlling for other variables. The SVR rates were not influenced by age, sex, the FIB index, genotype, baseline ALT, baseline HCV RNA, AFP, platelet count, hemoglobin, treatment regimen, or comorbidities.

Univariable and multivariable logistic regressions were also undertaken to identify the risk factors associated with the SVR in the entire cohort. In the univariate analysis, an absence of SVR12 was associated with treatmentexperienced patients $(\mathrm{OR}=7.68$; 95\% CI: $2.91-20.28$; $\mathrm{P}<0.001)$, compensated cirrhosis $(\mathrm{OR}=3.97 ; 95 \% \mathrm{CI}$ $=1.63-9.67 ; \mathrm{P}=0.002)$, decompensated cirrhosis $(\mathrm{OR}=12.03$; $95 \% \mathrm{CI}=3.55-40.71 ; \mathrm{P}<0.001)$, and genotype $3(\mathrm{OR}=4.89$; $95 \% \mathrm{CI}=1.56-15.31 ; \mathrm{P}=0.007)$. The factors independently associated with SVR12 included treatment-experience (aOR $=6.09 ; 95 \% \mathrm{CI}=1.76-21.07 ; \mathrm{P}=0.004)$, and decompensated cirrhosis $(\mathrm{aOR}=6.59 ; 95 \% \mathrm{CI}=1.20-36.28 ; \mathrm{P}=0.030)$. Factors such as age, sex, a history of HCC, the FIB index, genotype, baseline ALT, baseline AST, baseline HCV RNA, AFP, platelet count, hemoglobin, treatment regimen, or 
Table 3 Univariate and multivariate analysis of factors associated with SVR12 in $\geq 60$ years group

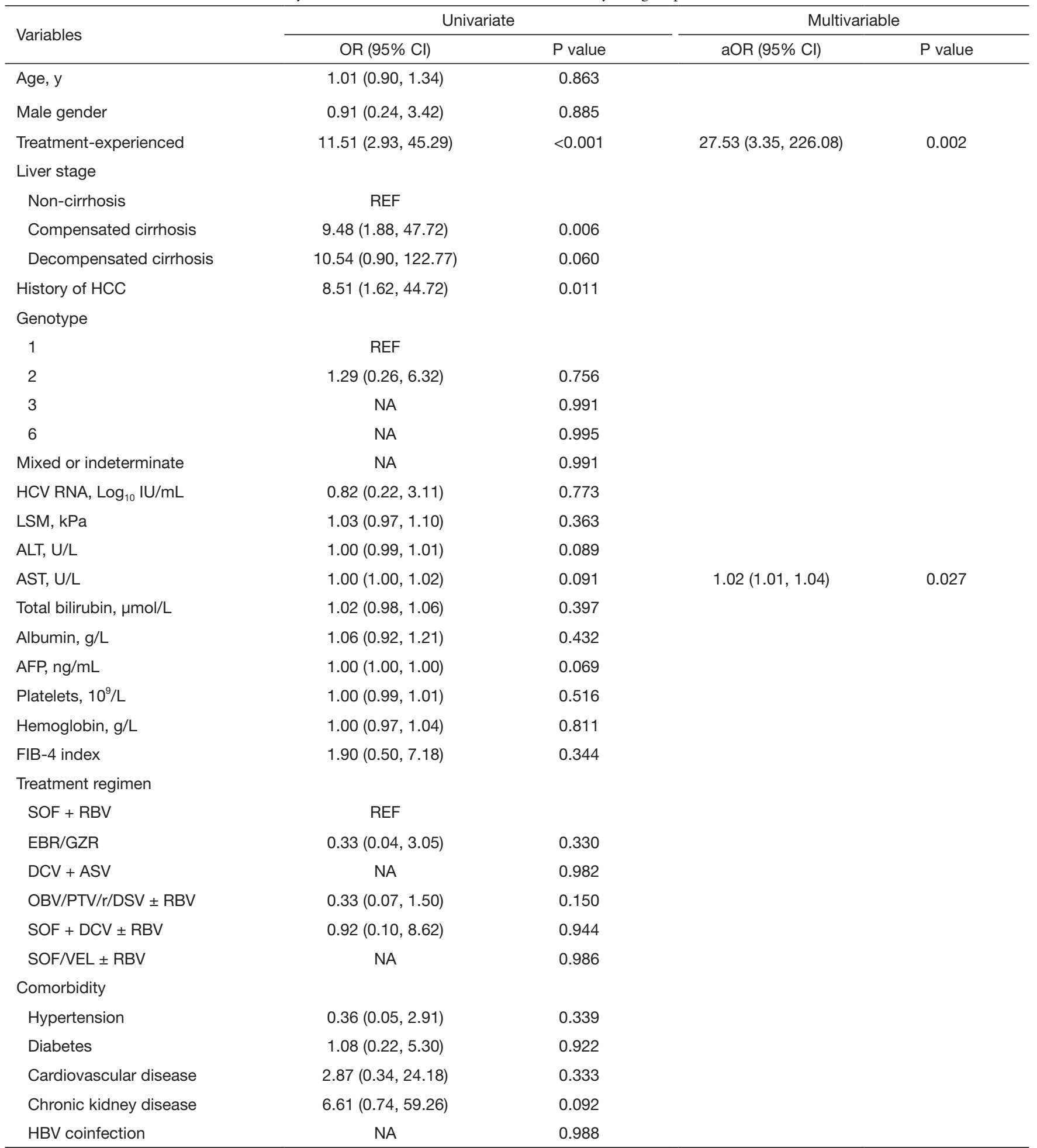

SVR12, sustained virologic response at 12 weeks post-treatment; OR, odds ratio; CI, confidence interval; aOR, adjusted odds ratio; HCC, hepatocellular carcinoma; HCV, hepatitis C virus; LSM, liver stiffness measurement; ALT, alanine aminotransferase; AST, aspartate aminotransferase; AFP, alpha-fetoprotein; FIB-4, Fibrosis-4; SOF sofosbuvir; RBV ribavirin; EBR, elbasvir; GZR, grazoprevir; DCV, daclatasvir; ASV, asunaprevir; OBV/PTV/r/DSV, ombitasvir/paritaprevir/ritonavir/dasabuvir; VEL, velpatasvir; HBV, hepatitis B virus; REF, reference category; NA, not available. 
Table 4 Safety of DAA regimen in older patients $\geq 60$ years $v s$. younger patients

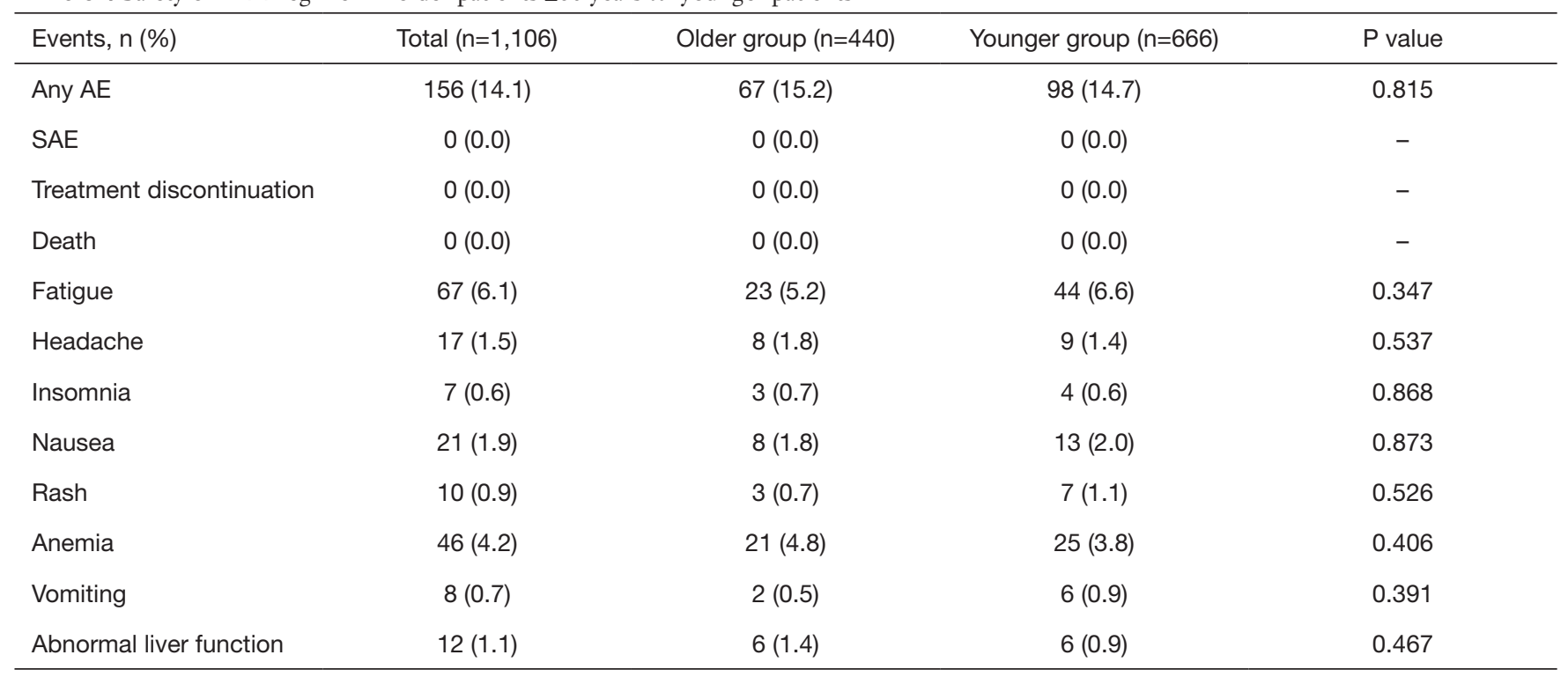

DAA, direct-acting antivirals; $A E$, adverse events; SAE, serious adverse events.

comorbidities did not affect the SVR12 rates (see Table S1).

\section{Safety and tolerability analysis}

Safety profiles are summarized in Table 4 . The proportion of AEs related to DAA therapy was similar in both older and younger patients $(15.2 \%$ vs. $14.7 \%, \mathrm{P}=0.815)$. The most commonly documented AEs were fatigue (6.1\%), anemia (4.2\%), nausea (1.9\%), and headaches (1.5\%). All of the AEs were mild and manageable. Not a single severe AE (leading to the discontinuation of treatment) was observed, and none of the patients died during the post-treatment follow-up period. However, it should be noted that 2 patients (both in the older group) developed newly detected HCC during the follow-up period. Our results suggest that older patients with chronic HCV infection can be safely treated with the currently available DAAs.

\section{Discussion}

To the best of our knowledge, this appears to be the first study to examine various brand DAA regimens in Chinese HCV patients aged $\geq 60$ years in a real-life setting. In China, the treatment of older HCV patients is an important issue due to the large number of $\mathrm{HCV}$-infected patients (based on the estimated prevalence) and China's aging population (30). Our results showed that younger and older HCV patients had comparable SVR results. Except for advanced cirrhosis and previous treatment failures, all 6 antiviral regimens achieved high rates of SVR12 in older patients affected by CHC, even in difficult-to-treat individuals with genotype 3. After controlling for potential confounding factors in multivariate models, we found that age was not an independent variable/predictor associated with SVR rates. Our study included a larger and more diverse population and had less strict eligibility criteria than those included in other randomized controlled trials, which is more representative of daily clinical practice.

The definition of old age varies across studies (3). Most developed Western countries define "old" individuals as those aged 65 years and above (31); however, this definition does not adapt well to China's situation. In line with the standard numerical criterion of the United Nations, China uses a cutoff is $60+$ years to refer to the elderly, which is equivalent to the legal retirement age (27). Previous studies on the efficacy of DAA therapy for older patients have mainly been based on different age groups, and the results are similarly highly efficient (17-19,32). Our study also showed that patients in three age subgroups (i.e., the age groups of $60-69,70-79$, and $\geq 80$ ) had excellent SVR results. Consequently, DAAs appear to have great promise in closing the cure-rate gap between different age groups.

Patients with comorbid medical conditions or those taking multiple medications are usually not involved in highly controlled clinical trials. Accordingly, the treatment outcomes for real-world prescribing may worsen in those 
specific sub-populations (33). Consistent with previous research $(17,34)$, our study reported a higher prevalence of comorbidities in older HCV patients, such as cardiovascular disease, hypertension, and diabetes. Thus, any selection of DAA regimens for older HCV patients should be made carefully. In addition, potential drug-drug interactions (DDIs) should not be underestimated or ignored, especially in the ritonavir-based regimen (35). Our results provide evidence that the presence of these comorbidities in older patients is not a barrier to achieving a SVR. After successful HCV treatment, further data need to be gathered to assess whether the health-related quality of life and life expectancy is improved in real-world clinical practices.

Chronic HCV infection is a leading cause of HCC, with an annual HCC incidence of $2-4 \%$ in cirrhotic patients (36). Most major international HCV cohorts have examined the risk of HCC in patients achieving SVR after DAA therapy. Collectively, SVR was associated with $~ 70 \%$ reduction in the risk of HCC. The effect was evident within 3-6 months and increased progressively with time $(36,37)$. The benefit of DAAs was evident across different demographic and clinical patients (36), different stages of cirrhosis $(36,37)$. The risk of HCC is reduced but not eliminated overtime after viral clearance by DAAs. Thus, routine HCC surveillance should continually be performed in patients with cirrhosis and advanced liver fibrosis who achieved DAA-induced SVR, as their absolute annual HCC risk remains high (37). In addition, cirrhosis and treatment failure were the commonest risk factors of HCC incidence (38). Other identified factors related to HCC development after DAA-induced SVR included male gender, older age, diabetes, alcohol consumption, and portal hypertension (38). As a result, long-term follow-up is necessary in the older HCV patients with greater risk of fibrosis and HCC development.

In this study, most AEs related to the 6 DAA regimens were mild and could be managed appropriately. Further, the rates of occurrence and the severity of treatment-related AEs were similar between older and younger patients. Thus, DAA regimens appear to be well-tolerated, even in patients aged $\geq 60$ years with $\mathrm{HCV}$ infection. Additionally, this study appears to provide evidence that the currently available DAAs are safe in such a population.

This study has several limitations. First, it was a retrospective study. Thus, sufficient data were not available in relation to some variables, including creatinine, international normalized ratio, body mass index, which may reduce the strength of our conclusions. Lager and future studies need to be conducted to verify the current results. Second, the assessment of potential DDIs was not performed. As the use of concomitant medications is more prevalent in older individuals, the risk of DDIs could make HCV treatment more challenging. DDIs become a critical concern for $\mathrm{HCV}$ patients with comorbidities that require concomitant medications, which can change the drug's exposure and are related to decreased efficacy, adverse reactions, and suboptimal adherence (39); therefore, confirmation of DDIs before prescribing DAAs was recommended. Health providers should pay more attention to potential DDIs when selecting DAA regimens for HCV patients. Third, pre-treatment resistanceassociated substitutions (RASs) in the non-structural protein (NS) 5A/NS5B regions were not tested; the RASs were reported to be related to the virologic failure of DAA therapies (40). However, several studies have shown that baseline NS5A RAS levels of Chinese CHC patients were relatively low and, most likely, did not reduce treatment efficacy $(41,42)$. Additionally, the lack of data on dynamic changes of ALT, AFP, and Fibroscan ${ }^{\circledR}$ results over time limits our understanding of the effects of DAA therapy on biochemical parameters and the liver FIB marker (FIB-4). A recent study (16) reported the same pattern of dynamic changes in undetectable HCV RNA rate and ALT normalization in older patients by direct comparison with younger patients. The older patients displayed a higher rate of pre-treatment AFP elevation but delayed and lower AFP normalization. High post-treatment AFP level is the risk factor predictive of HCC development post SVR (38). Therefore, periodical surveillance of metabolic profiles and fibrosis status post SVR in older patients should be adopted as a routine clinical practice. Last, retrospective cohort studies may introduce sample selection bias and information bias or misclassification; accordingly, specific caution for errors due to bias and confounding should be taken.

To conclude, our study represents one of the largest observational studies of older HCV patients exposed to available brand DAAs in China. The results provide valuable insights into the efficacy and generalizability of DAAs in a larger heterogeneous population.

\section{Acknowledgments}

We would like to thank the participants for their contributions to this study. 
Funding: This work was supported by the Key Project of Science and Technology Fund of Tianjin Health Commission (No. 2014KR03), and the 13th Five-year National Major Project for HIV/AIDS and Hepatitis B Control and Prevention, Chinese Ministry of Science and Technology (No. 2017ZX10202102005004).

\section{Footnote}

Reporting Checklist: The authors have completed the STROBE reporting checklist. Available at http://dx.doi. org/10.21037/atm-21-1297

Data Sharing Statement: Available at http://dx.doi. org/10.21037/atm-21-1297

Conflicts of Interest: All authors have completed the ICMJE uniform disclosure form (available at http://dx.doi. org/10.21037/atm-21-1297). Dr. PM reports grants from the Key Project of Science and Technology Fund of Tianjin Health Commission (No. 2014KR03), and the 13th Five-Year National Major Project for HIV/AIDS and Hepatitis B Control and Prevention, Chinese Ministry of Science and Technology (No. 2017ZX10202102005004) during the conduct of the study. The other authors have no conflicts of interest to declare.

Ethical Statement: The authors are accountable for all aspects of the work in ensuring that questions related to the accuracy or integrity of any part of the work are appropriately investigated and resolved. The study was conducted in accordance with the Declaration of Helsinki (as revised in 2013). This study was reviewed and approved by the Tianjin Second People's Hospital Institutional Review Board (No. 2018-8), which is a leading research institute. Written and informed consent was provided by each of the recruited participants.

Open Access Statement: This is an Open Access article distributed in accordance with the Creative Commons Attribution-NonCommercial-NoDerivs 4.0 International License (CC BY-NC-ND 4.0), which permits the noncommercial replication and distribution of the article with the strict proviso that no changes or edits are made and the original work is properly cited (including links to both the formal publication through the relevant DOI and the license). See: https://creativecommons.org/licenses/by-nc-nd/4.0/.

\section{References}

1. Polaris Observatory HCVC. Global prevalence and genotype distribution of hepatitis $\mathrm{C}$ virus infection in 2015: a modelling study. Lancet Gastroenterol Hepatol 2017;2:161-76.

2. Gao Y, Yang J, Sun F, et al. Prevalence of Anti-HCV Antibody Among the General Population in Mainland China Between 1991 and 2015: A Systematic Review and Meta-analysis. Open Forum Infect Dis 2019;6:ofz040.

3. Huang CF, Yu ML. Treating hepatitis $C$ in the elderly: pharmacotherapeutic considerations and developments. Expert Opin Pharmacother 2017;18:1867-74.

4. Liu Z, Yang Q, Shi O, et al. The epidemiology of hepatitis B and hepatitis C infections in China from 2004 to 2014: An observational population-based study. J Viral Hepat 2018;25:1543-54.

5. Yu ML, Huang CF, Yeh ML, et al. Time-Degenerative Factors and the Risk of Hepatocellular Carcinoma after Antiviral Therapy among Hepatitis C Virus Patients: A Model for Prioritization of Treatment. Clin Cancer Res 2017;23:1690-7.

6. Ogawa E, Nomura H, Nakamuta M, et al. Development of Hepatocellular Carcinoma by Patients Aged 75-84 with Chronic Hepatitis C Treated with Direct-acting Antivirals. J Infect Dis 2020. [Epub ahead of print]. doi: 10.1093/ infdis/jiaa359.

7. Chou R, Dana T, Fu R, et al. Screening for Hepatitis C Virus Infection in Adolescents and Adults: Updated Evidence Report and Systematic Review for the US Preventive Services Task Force. JAMA 2020. [Epub ahead of print]. doi: 10.1001/jama.2019.20788.

8. Kaplan DE. Hepatitis C Virus. Ann Intern Med 2020;173:ITC33-ITC48.

9. US Preventive Services Task Force, Owens DK, Davidson $\mathrm{KW}$, et al. Screening for Hepatitis C Virus Infection in Adolescents and Adults: US Preventive Services Task Force Recommendation Statement. JAMA 2020;323:970-5.

10. Sarrazin C. Treatment failure with DAA therapy: importance of resistance. J Hepatol 2021;74:1472-82.

11. European Association for the Study of the Liver. Electronic address: easloffice@easloffice.eu; Clinical Practice Guidelines Panel: Chair:; EASL Governing Board representative:; Panel members.. EASL recommendations on treatment of hepatitis C: Final update of the series 2 . J Hepatol 2020;73:1170-218.

12. Panel A-IHG. Hepatitis C Guidance 2018 Update: AASLD-IDSA Recommendations for Testing, Managing, 
and Treating Hepatitis C Virus Infection. Clin Infect Dis 2018;67:1477-92.

13. Omata M, Kanda T, Wei L, et al. APASL consensus statements and recommendation on treatment of hepatitis C. Hepatol Int 2016;10:702-26.

14. Yanny B, Saab S, Durazo F, et al. Eight-Week Hepatitis C Treatment with New Direct Acting Antivirals Has a Better Safety Profile While Being Effective in the TreatmentNaive Geriatric Population Without Liver Cirrhosis and Hepatitis C Virus-RNA < 6 Million IU/mL. Dig Dis Sci 2018;63:3480-6.

15. Ascione A, De Luca M, Melazzini M, et al. Safety and efficacy of ombitasvir/paritaprevir/ritonavir/dasabuvir plus ribavirin in patients over 65 years with $\mathrm{HCV}$ genotype 1 cirrhosis. Infection 2018;46:607-15.

16. Huynh T, Hu KQ. Excellent Safety and Sustained Virologic Response to Direct-Acting Antivirals Treatment in HCV-Infected Geriatric Patients: A Real-World Data. Dig Dis Sci 2021;66:1327-34.

17. Pan CQ, Gayam V, Rabinovich C, et al. Efficacy of DirectActing Antivirals for Chronic Hepatitis C in a Large Cohort of Older Adults in the United States. J Am Geriatr Soc 2020;68:379-87.

18. Mazzarelli C, Considine A, Childs K, et al. Efficacy and Tolerability of Direct-Acting Antivirals for Hepatitis C in Older Adults. J Am Geriatr Soc 2018;66:1339-45.

19. Kinoshita A, Koike K, Mizuno Y, et al. Efficacy and safety of glecaprevir/pibrentasvir in patients with hepatitis $\mathrm{C}$ virus infection aged $>/=75$ years. Geriatr Gerontol Int 2020;20:578-83.

20. Ogawa E, Furusyo N, Nomura H, et al. Effectiveness and safety of sofosbuvir plus ribavirin for HCV genotype 2 patients 65 and over with or without cirrhosis. Antiviral Res 2016;136:37-44.

21. Elbaz T, Abdo M, Omar H, et al. Efficacy and safety of sofosbuvir and daclatasvir with or without ribavirin in elderly patients with chronic hepatitis $\mathrm{C}$ virus infection. J Med Virol 2019;91:272-7.

22. Trifan A, Stanciu C, Gheorghe L, et al. Efficacy and safety of paritaprevir/ritonavir, ombitasvir, and dasabuvir with ribavirin for the treatment of $\mathrm{HCV}$ genotype $1 \mathrm{~b}$ compensated cirrhosis in patients aged 70 years or older. Medicine (Baltimore) 2017;96:e9271.

23. Zhang P, Guo R, Lian J, et al. Unblocking Barriers of Access to Hepatitis C Treatment in China: Lessons Learned from Tianjin. Ann Glob Health 2020;86:36.

24. Xia H, Lu C, Wang Y, et al. Efficacy and Safety of DirectActing Antiviral Therapy in Patients With Chronic
Hepatitis C Virus Infection: A Real-World Single-

Center Experience in Tianjin, China. Front Pharmacol 2020;11:710.

25. Chinese Society of Hepatology; Chinese Society of Infectious Diseases, Chinese Medical Association.

Guidelines for the prevention and treatment of hepatitis C (2019 version). Zhonghua Gan Zang Bing Za Zhi 2019;27:962-79.

26. WHO. Ageing. World Health Organization. 2020. Available online: https://www.who.int/health-topics/ ageing. Accessed 1 June 2020.

27. China TNPsCotPsRo. Elderly Rights Guarantees Law in the people's Republic of China. The National People's Congress of the People's Republic of China. 2018. Available online: http://www.npc.gov.cn/npc/c30834/2019 01/47231a5b9cf94527a4a995bd5ae827f0.shtml. Accessed 1 June 2020.

28. Forman DE, Berman AD, McCabe CH, et al. PTCA in the elderly: the "young-old" versus the "old-old". J Am Geriatr Soc 1992;40:19-22.

29. Cancer Therapy Evaluation Program CTCAE V5.0. USA: National Institutes of Health. 2017. Available online: https://ctep.cancer.gov/protocolDevelopment/electronic_ applications/docs/CTCAE_v5_Quick_Reference_8.5x11. pdf. Accessed 1 June 2020.

30. Wei L, Lok AS. Impact of new hepatitis C treatments in different regions of the world. Gastroenterology 2014;146:1145-50.e1-4.

31. Villani R, Monami M, Di Cosimo F, et al. Directacting antivirals for $\mathrm{HCV}$ treatment in older patients: A systematic review and meta-analysis. J Viral Hepat 2019;26:1249-56.

32. Su F, Beste LA, Green PK, et al. Direct-acting antivirals are effective for chronic hepatitis $\mathrm{C}$ treatment in elderly patients: a real-world study of 17487 patients. Eur J Gastroenterol Hepatol 2017;29:686-93.

33. Dultz G, Muller T, Petersen J, et al. Effectiveness and Safety of Direct-Acting Antiviral Combination Therapies for Treatment of Hepatitis C Virus in Elderly Patients: Results from the German Hepatitis C Registry. Drugs Aging 2018;35:843-57.

34. Conti F, Brillanti S, Buonfiglioli F, et al. Safety and efficacy of direct-acting antivirals for the treatment of chronic hepatitis $\mathrm{C}$ in a real-world population aged 65 years and older. J Viral Hepat 2017;24:454-63.

35. Schulte B, Wubbolding M, Marra F, et al. Frequency of Potential Drug-Drug Interactions in the Changing Field of HCV Therapy. Open Forum Infect Dis 2020;7:ofaa040. 
36. Singal AG, Lim JK, Kanwal F. AGA Clinical Practice Update on Interaction Between Oral DirectActing Antivirals for Chronic Hepatitis C Infection and Hepatocellular Carcinoma: Expert Review.

Gastroenterology 2019;156:2149-57.

37. Calvaruso V, Craxi A. Hepatic benefits of HCV cure. J Hepatol 2020;73:1548-56.

38. Hsu SJ, Yang SS, Kao JH. Risk of hepatocellular carcinoma development after hepatitis $\mathrm{C}$ virus eradicated by directacting antivirals: Fact or fiction? J Formos Med Assoc 2020;119:3-11.

39. Garrison KL, German P, Mogalian E, et al. The DrugDrug Interaction Potential of Antiviral Agents for the Treatment of Chronic Hepatitis C Infection. Drug Metab Dispos 2018;46:1212-25.

Cite this article as: Xia $\mathrm{H}$, Zhang $\mathrm{Y}$, Zaongo SD, Liang J, Gong X, Hu Y, Ma P, Wang F. Direct-acting antiviral treatments display excellent outcomes even in older HCVinfected patients at increased risk of fibrosis. Ann Transl Med 2021;9(10):847. doi: 10.21037/atm-21-1297
40. Iio E, Shimada N, Abe H, et al. Efficacy of daclatasvir/ asunaprevir according to resistance-associated variants in chronic hepatitis $\mathrm{C}$ with genotype 1. J Gastroenterol 2017;52:94-103.

41. Wei L, Xie Q, Hou JL, et al. Sofosbuvir plus ribavirin with or without peginterferon for the treatment of hepatitis $\mathrm{C}$ virus: Results from a phase $3 \mathrm{~b}$ study in China. J Gastroenterol Hepatol 2018;33:1168-76.

42. Wei L, Xie Q, Hou JL, et al. Ledipasvir/sofosbuvir for treatment-naive and treatment-experienced Chinese patients with genotype $1 \mathrm{HCV}$ : an open-label, phase $3 \mathrm{~b}$ study. Hepatol Int 2018;12:126-32.

(English Language Editor: L. Huleatt) 


\section{Supplementary}

Table S1 Univariate and multivariate analysis of factors associated with SVR12 in the entire cohort

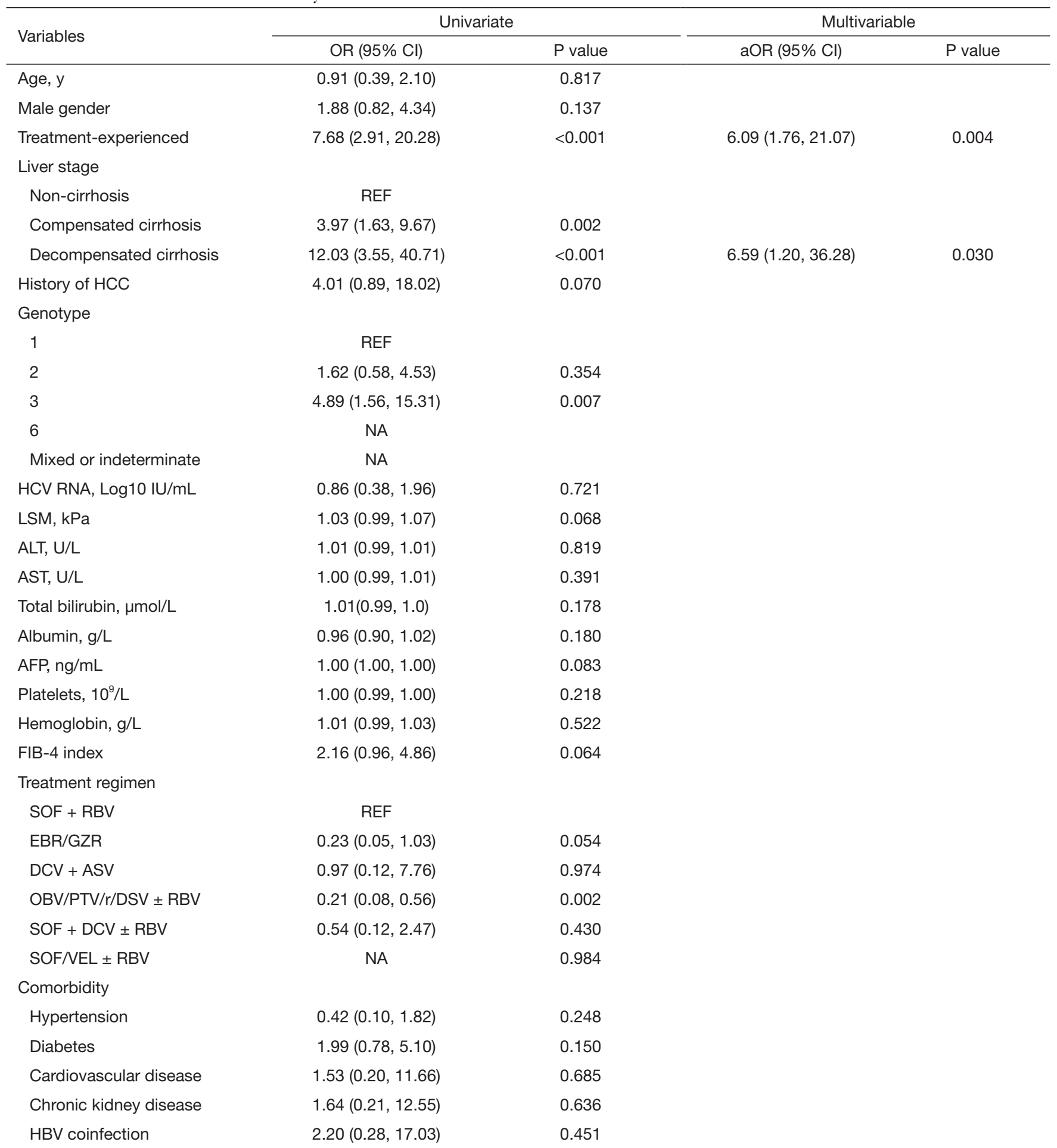

SVR12, sustained virologic response at 12 weeks post-treatment; OR, odds ratio; Cl, confidence interval; aOR, adjusted odds ratio; HCC, hepatocellular carcinoma; HCV, hepatitis C virus; LSM, liver stiffness measurement; ALT, alanine aminotransferase; AST, aspartate aminotransferase; AFP, alpha-fetoprotein; FIB-4, Fibrosis-4; SOF, sofosbuvir; RBV, ribavirin; EBR, elbasvir; GZR, grazoprevir; DCV daclatasvir; ASV, asunaprevir; OBV/PTV/r/DSV, ombitasvir/paritaprevir/ritonavir/dasabuvir; VEL, velpatasvir; HBV, hepatitis B virus; REF, reference category; NA, not available. 